\title{
Nanoscale Calorimetry Reveals Higher Stability of Cholesterol Induced Nanoscale Domains in Lipid Bilayers
}

\author{
Georg E. Fantner ${ }^{1}$, Blake W. Erickson ${ }^{1}$ \\ ${ }^{1}$ École polytechnique federal de Lausanne, Interfaculty Institute for Bioengineering, Lausanne, \\ Switzerland
}

Membranes of all cells contain a significant fraction of phospholipids arranged in a self-assembled bilayer. This lipid bilayer forms a robust barrier to ions, proteins and other molecules, and separates the inside of the cell from the outside (plasma membrane), or compartmentalizes the cells (mitochondria). Embedded in the lipid bilayers are a large variety of glycoproteins, globular proteins, and membrane channels[1]. The way these molecules perform their functions is strongly dependent on their location and the mechanical and chemical properties of the lipid environment around them. In cellular membranes, it is believed that lipid rafts (small lipid domains enriched in cholesterol) act to hold membrane proteins together in a dynamic fashion[2,3]. The difference in the stability between the lipid rafts and the fluid bilayer is believed to be very small.

The stability and rigidity of lipid bilayers depends on the type of lipid, temperature, and the presence of cholesterol amongst other factors. Many naturally occurring lipids exhibit phase transition around room temperature, which drastically alters the membrane's stability. In nature, lipid membranes consist of mixtures of many different types of lipids, proteins, and other molecules. The inhomogeneity of these membranes result in spatially distributed differences in stability[4-6]. Here we present a method to measure this inhomogeneity in the stability of lipid membranes with nanometer resolution, by using atomic force microscopy induced phase transition.

We use a combination of global heating[7] and local mechanical work to induce local phase transitions in model lipid membranes. In a modified AFM, we control the local temperature using laser heating, and locally apply mechanical energy using Peak Force Quantitative Nanoscale Mapping. By varying these two parameters we can locally induce phase transitions.

Using this method, we have revealed a region of increased stability at the domain boundaries in a binary mixed system of the phospholipids, DLPC and DPPC, in the absence of cholesterol. In the presence of cholesterol, additional nanoscale domains exist inside the predominantly DPPC patches of the mixed lipid bilayer. These domains have increased stability and sizes comparable to those postulated for lipid rafts in cell membranes.

This technique provides the first direct method to image and investigate stable domains on the length scale of lipid rafts. We believe that this technique will provide complementary information to fluorescence[8] and NMR[9] techniques which have been the only indirect methods available to date.

[1] K. Simons, W.L.C. Vaz, Annu. Rev. Biophys. Biomol. Struct. 33 (2004) 269.

[2] D. Lingwood, H.-J. Kaiser, I. Levental, K. Simons, Biochem. Soc. Trans. 37 (2009) 955.

[3] D. Lingwood, K. Simons, Science 327 (2010) 46.

[4] G.W. Feigenson, J.T. Buboltz, Biophys. J. 80 (2001) 2775.

[5] T. Baumgart, S.T. Hess, W.W. Webb, Nature 425 (2003) 821. 
[6] S.L. Veatch, S.L. Keller, Biophys. J. 85 (2003) 3074.

[7] C.D. Blanchette, C.A. Orme, T. V Ratto, M.L. Longo, Langmuir 24 (2008) 1219.

[8] J. Korlach, P. Schwille, W.W. Webb, G.W. Feigenson, Proc. Natl. Acad. Sci. U. S. A. 96 (1999) 8461.

[9] S.L. Veatch, I. V Polozov, K. Gawrisch, S.L. Keller, Biophys. J. 86 (2004) 2910.

The authors acknowledge funding from the Swiss National Science Foundation under award number 205321_134786, the European Union's Seventh Framework Program FP7/2007-2011 under grant agreement n ${ }^{\circ} 286146$, and the European Research Council project NaMic.

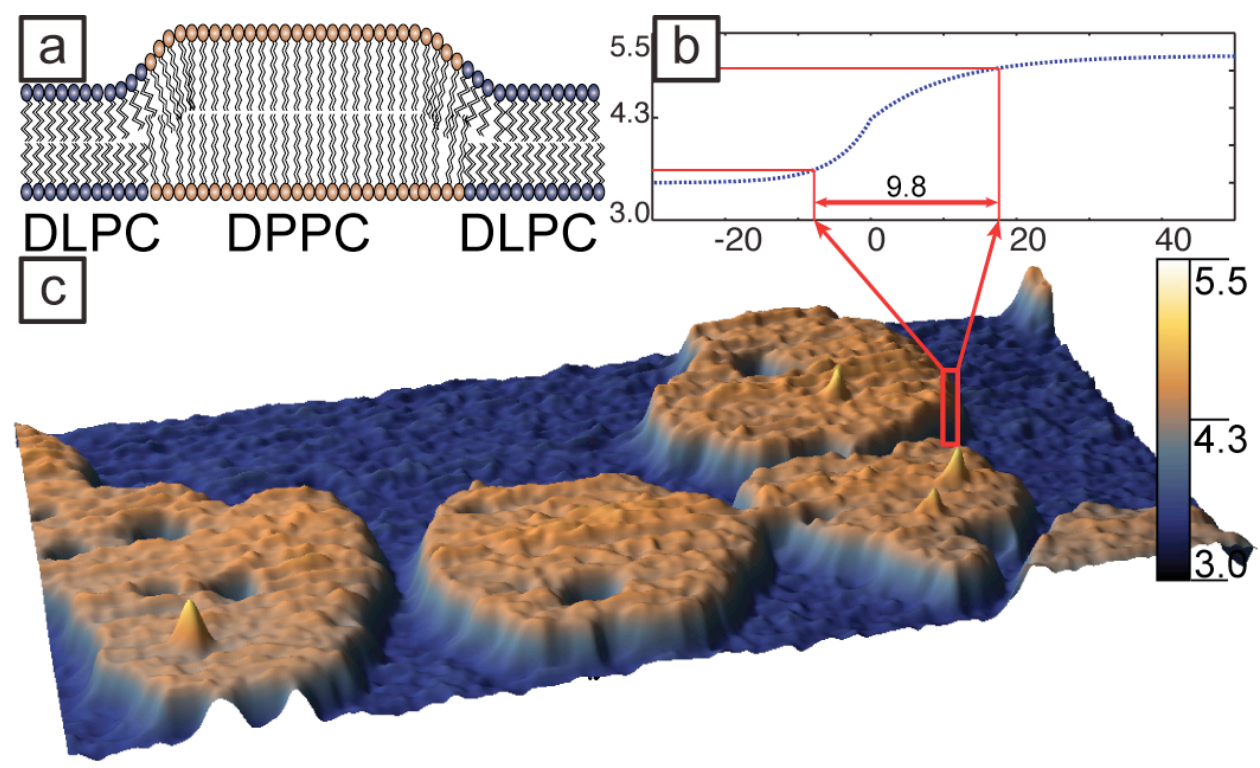

Figure 1. Lipid bi-layer of DLPC and DPPC. A) Schematic showing the origin of the phase segregation and the height mismatch. B) Predicted size of the interface region (REF). C) AFM image of the phase segregated layer.
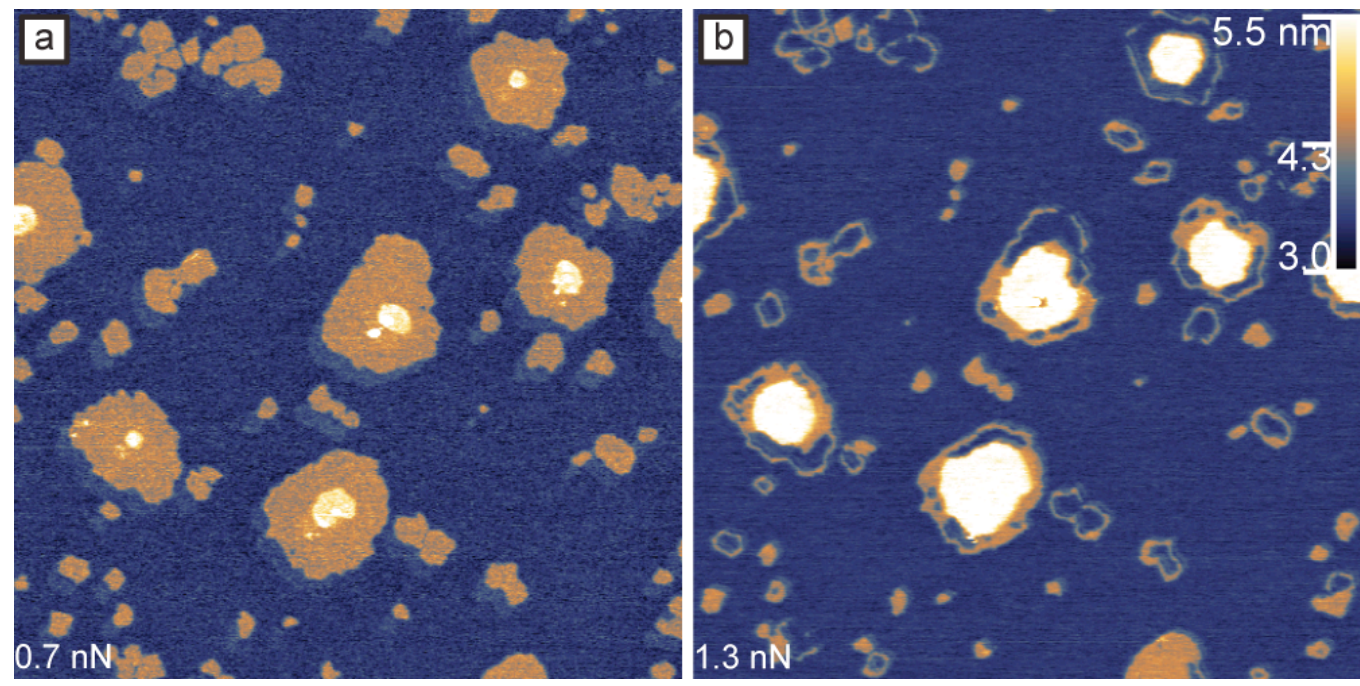

Figure 2. Mixed lipid bilayer. A) imaged at low forces. B) Energy added by the AFM tip locally induces a phase transition in the "bulk" DPPC, leaving the higher stability border region intact. 